\title{
Conductance and application of organic molecule pairs as nanofuses
}

\author{
Salvador Rodríguez-Bolívar, ${ }^{1, *}$ Francisco M. Gómez-Campos, ${ }^{1, \dagger}$ Luis Álvarez de Cienfuegos, ${ }^{2, \dagger}$ Noelia Fuentes, ${ }^{2}$ \\ Diego J. Cárdenas, ${ }^{3}$ Elena Buñuel, ${ }^{3}$ Juan E. Carceller, ${ }^{1}$ Andrés Parra, ${ }^{2}$ and Juan M. Cuerva ${ }^{2, \S}$ \\ ${ }^{1}$ Department of Electronic and Computer Technology, Universidad de Granada, Campus Fuentenueva s/n, E-18071, Granada, Spain \\ ${ }^{2}$ Department of Organic Chemistry, Universidad de Granada, Campus Fuentenueva s/n, E-18071, Granada, Spain \\ ${ }^{3}$ Department of Organic Chemistry C-I, Universidad Autónoma de Madrid, Cantoblanco E-28049 Madrid, Spain
}

(Received 20 August 2010; revised manuscript received 22 January 2011; published 25 March 2011)

\begin{abstract}
We propose that a pair of organic molecules can mimic the behavior of a macroscopic fuse at nanoscale, one component of the pair being the on state and the other the off state. For this task we make use of density-functional theory to calculate the physical properties of selected molecules, which have also been synthesized by our team. By this means we obtain the transmission spectra and the current of the proposed devices, which allows us to compare the behavior of the on and off states. Of particular interest is the on/off switch ratios, defined as the current ratios of the on and off structures at the corresponding bias voltage. In a first stage, we examine the best linker between the device and the electrode for high on/off switch ratios. Once this is determined, we test the influence of the electron richness of the system to provide a high on/off switch ratio. The entire analysis is also supported by the molecular projected self-consistent Hamiltonian, which provides a good way of understanding the molecular behavior. All the calculations support that interesting on/off switch ratios of two orders of magnitude could be obtained with these prototypical nanofuses.
\end{abstract}

DOI: 10.1103/PhysRevB.83.125424

PACS number(s): 72.80.Le, 73.63.Rt, 71.15.Mb

\section{INTRODUCTION}

Electron transport through conjugated organic molecules is a central issue in molecular electronics and in material science on the nanoscale. ${ }^{1-14}$ These molecules have critical properties for high conductance, such as small highest occupied molecular orbital (HOMO)-lowest unoccupied molecular orbital (LUMO) gaps and fully delocalized $\pi$ systems. They can be used, for example, to connect electron reservoirs at the molecular level. In fact, in nature there are excellent examples of conjugated molecules facilitating the electronic connection between metallic centers in enzymatic systems. ${ }^{15-17}$ Control of the flow of electrons between the two ends of molecular conductors is valuable in many scientific fields and may open up the possibility of simulating the behavior of standard circuitry at the molecular level. ${ }^{18,19}$

Some successful approaches to molecular switches based on metallic complexes have been reported using a difference of voltage to promote a change in the oxidation state of the metallic center. If we define the on state as the state of maximum conductance and the off state as the state of minimum conductance, they work in the offlon sense when the stimulus is increased, and consequently are voltage dependent. Moreover, they are reversible, ${ }^{20}$ i.e., these switches change their conductivity when a specific voltage is applied but recover their initial features when the voltage is removed. The development of systems working in the on/off sense with an irreversible behavior to the voltage, mimicking a macroscopic fuse, is much less extensive. Ideally, when situated in a molecular circuit, these could (i) detect an overvoltage, thus protecting complex electronic systems, (ii) trigger other processes in a controlled manner, and (iii) act as binary components of read-only memories (ROMs). ${ }^{21}$

These factors prompted us to investigate this class of electronic device. In principle, a simple molecular fuse has to mimic the behavior of its macroscopic analogs.
Therefore we have imposed the following key features for an acceptable molecular fuse: (i) an easy integration into other previously reported "molecular wires," (ii) to be able to drop its conductance at a desirable voltage with a high ratio between the conductivity of the on and off states, (iii) in some applications it should be desirable to be "switched" to the on state again in a controlled manner using some chemical or physical process, (iv) to be chemically stable in the off state at high voltage and, (v) to keep the same geometry and length in the on and off states in order to preserve the global structure of the system. This is an essential feature because it prevents any mechanical stress on the system when the switching process takes place. For example, photoswitches based on dithienylethene derivatives have the drawback that despite having excellent switching properties, they undergo changes in length and geometry during the switching event and a subsequent reorganization in the junction occurs to reach the final equilibrium state. $^{22}$

With these requirements in mind we focused our attention on $p$-dialkoxybenzenes (I), which can be electrochemically oxidized to quinone bisketals (II). In this case, two oxygen atoms must be incorporated to the structure (Fig. 1)..$^{23,24}$ The source of such oxygen atoms can be diverse. Thus, for example, in homogeneous environments oxygenated solvents or additives, such as methanol, are usually employed as an oxygen-atom source. Another solution is to incorporate the oxygen atom to the molecular framework at the expense of increasing the complexity of the global system. Nevertheless, such more complex "molecular fuses" are required for solidstate devices, owing to the absence of external sources of appropriate oxygen atoms. It is also worth noting that in the global reaction two protons are also lost into the homogeneous environment. In solid-state devices the fate of the hydrogen atoms is open to debate. It has been suggested that they 
<smiles>Oc1ccc(O)cc1</smiles>

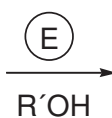

I<smiles>OCCOc1ccc(OCCO)cc1</smiles><smiles>[R]OC1([R])C=CC([R])([R])C=C1</smiles>

II

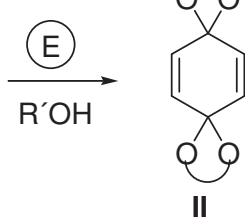

Homogeneous enviroment

\section{Solid state}

\section{MOLECULAR FUSES}

In this work we have focused our attention on the capabilities for electron transport through molecular junctions of structures I and II. Although now they are experimentally available and their conductivity could be measured, such measures are usually difficult to carry out and a prior selection of the molecular targets is mandatory. ${ }^{39,40}$

Our working hypothesis is summarized in Fig. 2: The initial conductor system I can be electrochemically transformed into the insulator system II by the application of a suitable voltage in the presence of an appropriate oxygen source. Although for the on/off switch event development of charge in the molecule derived from hole-based electron transport (ET) is required, we have focused our study on the coherent ET mechanism, especially under low bias, which would be the usual working voltage for the device.

Theoretical approaches to molecular conduction based on density-functional theory (DFT) are now available ${ }^{41,42}$ and can be used to discriminate between the structures with the best profiles and to understand the mechanism of ET through molecular conductors. The main limitation is that the experimental data and the theoretical predictions are not always in good agreement ${ }^{43,44}$ due to multiple factors, such as geometric fluctuations in molecule-electrode interfaces, ${ }^{45}$ structurally nonideal electrode surface interactions, ${ }^{46}$ fast modifications of the internal geometry of the molecule, ${ }^{47}$ solvent-molecule interactions, ${ }^{48,49}$ or an underestimation of the band gap. ${ }^{50,51}$ Nevertheless, we are mainly interested in the evaluation of the on/off switch ratios. Therefore we think that by maintaining the same computational parameters (functional, basis set, energy shift, Monkhorst-Pack grid, and pseudopotentials) in both systems the theoretical results may be comparable. stable and not transient states and could be synthesized and characterized independently and their corresponding properties measured. In fact, we have recently prepared some of these structures and proved experimentally their main hypothesized characteristics. $^{38}$

Therefore, in principle, I/II pairs could be considered as a prototype of a molecular fuse. Nevertheless, some relevant issues should first be analyzed to prove their suitability. In particular, information about the on/off switch ratios, defined as the current ratios of the on and off structures at the corresponding bias voltage, is mandatory for this device. In this work we have studied this aspect computationally
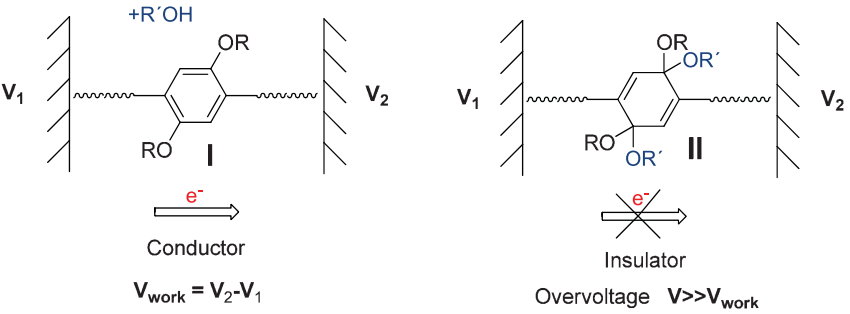

FIG. 2. (Color online) Working hypothesis. 
From a practical point of view, the on/off switch ratio should be as high as possible. In the literature, different experimental switching ratios can be found: $2,{ }^{52} 25,{ }^{53,54} 100,{ }^{55} 250$ (Ref. 56) $(1 \mathrm{~V}),{ }^{57} 302,{ }^{58} 500(0.55 \mathrm{~V}),{ }^{59} 1000,{ }^{60}$ or even examples in which the off state is literally "unplugged" to maximize the ratio. ${ }^{61}$ However, the above described switching processes usually take place only from the off state to the on state when the external factor is enabled, and change to the off state again when that factor is removed. They are different from those studied in this paper because our proposed devices are able to work in the opposite on/off sense when increasing the voltage, and can also be maintained in the desirable state when the voltage is removed. To study the magnitude of on/off switch ratios, in this paper we have focused on the effects of two key parameters: (a) the nature of the linkers and (b) the effect of the substituents on the dialkoxybenzene moiety.

In Fig. 3(a) two different processes are shown to convert structure I to structure II. The one shown on the right needs the oxygen atom to be incorporated from an external source. However, for many solid-state devices no exogenous oxygen source is available and therefore more elaborate "molecular fuses" are required. The straightforward solution is the insertion into the structure of a pedant group including the required oxygen atom ${ }^{62}$ as shown in the process on the left of this figure.

Based on these premises, we have selected some specific devices from those with structures shown in Fig. 3(b). They present $p$-terphenyl (TP) and expanded $p$-terphenyl structures

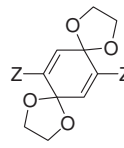

$\mathrm{R}=\mathrm{CH}_{2} \mathrm{CH}_{2} \mathrm{OH}$
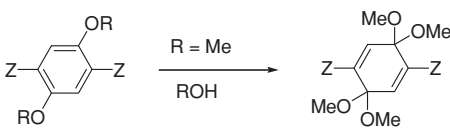

(a)

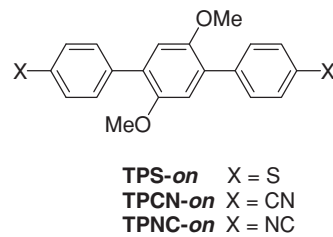

TPNC-on $X=N C$

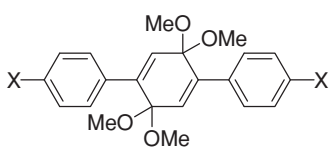

TPS-off1 $X=S$ TPCN-off1 $X=C N$ TPNC-off1 $X=N C$

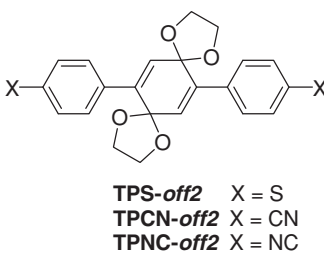

(b)

FIG. 3. (a) Processes to convert structure I to structure II. (b) Definition of the structures to be simulated.
(ETP). These kinds of structures are highly valuable in fundamental studies of molecular conductivity, ${ }^{63,64}$ their linear and rigid arrangements being highly appreciated in the field.

(E)TP-on are the on devices while (E)TP-off are their off states. The conductivity of the on states is mainly determined by the aromatic core of conducting structures I and a similar behavior is expected regardless of the substituents in the pedant alkoxy side chain. Therefore for simplicity we have also considered TP-on as the on states of the off devices TP-off 2 when calculating the on/off switch ratios. Furthermore, it is known that the torsion angle plays a major role in the conductivity of the system. ${ }^{65}$ Thus structures TP-offl and TP-off 2 can be used to determine the influence of the torsion angle on the conductivity. Similar reasoning has been used for the ETP-on devices and the off states ETP-off.

The presence of a group acting as linker between the electrodes and the molecule is indispensable in experimental electrode-molecule-electrode heterostructures. ${ }^{66}$ The nature of such linker in contact with the metallic electrode and its ability to hybridize molecule and electrode electronic states determine the regime of electron transport. ${ }^{67}$ For high conductivity, a strong-coupling regime is required. In this case, it has been described that linkers based on nitrogen, ${ }^{68-75}$ sulphur, ${ }^{76-82}$ phosphorus, ${ }^{83}$ or carbon ${ }^{84}$ can be used to carry out measurements of conductivity values.

To carry out the study of the linkers, we selected TP structures to calculate the on/off switch ratios of our proposed molecular fuses. Thus we included linkers with different electronic properties: (i) a sulphur-based electron-rich anchoring group (TPS), and (ii) two electron-poor nitrogen-based anchoring groups with different affinities to metals, such as nitrile (weak) TPCN and isonitrile (strong) TPNC. ${ }^{85,86}$ As mentioned above, a problem in the study of TP structures is that they are nonplanar and the corresponding torsion angle between the aromatic rings is a key parameter in the conduction through such molecules. We have taken this effect into account when studying the conductivity through the conformations of minimum energy determined by our DFT calculations. This is a crucial point for us since we are also interested in building up the corresponding devices to validate our predictions and thus we must assume the most realistic situation in the calculation.

Once the linker analysis was made, in order to study the effect of substituents in the core aromatic ring, we selected ETP structures, which are almost planar in their ground states. The spacers between the aromatic rings ensure that the presence of the substituents does not significantly affect the torsion angle of the device and such effects can be almost eliminated.

\section{COMPUTATIONAL METHODOLOGY}

One common approach in this kind of study is to build a conceptual device in which molecules are anchored between metallic electrodes in a sandwich-type junction. This configuration simulates the frequently used approach of scanning probe microscopy to measure molecular conductivity. In this area, there is abundant literature about systems using gold for metallic electrodes in both theoretical and experimental studies. For simplicity and to facilitate an experimental verification of our predictions, we selected bulk gold with 
$\mathrm{Au}(111)$ surfaces as electrodes throughout our study. Instead of simulating the $\mathrm{Au}$ electrodes with thousands of atoms, we simulated the Au electrode surfaces using a $(4 \times 4)$ cell with periodic boundary conditions. From this, we defined a supercell consisting of two layers of 32 and 48 atoms, respectively, on both sides of the scattering region. The size of the supercell ensures that there is no significant interaction between the molecules of the next neighboring supercells.

Gas phase TP and ETP structures were previously optimized by Gaussian 03 at the DFT level, using the B3LYP hybrid functional and the 6-31G(d,p) basis set for all atoms. They were then joined to these Au electrodes. However, the nature of the junction depends on the nature of the linker. The sulphur-Au junction has been extensively studied. Although the nature of the gold-sulphur bond is not clear, some recent advances have been achieved, ${ }^{87,88}$ and many theoretical studies predict that "triangle" (interaction with two Au atoms) and "pyramid" (interaction with three $\mathrm{Au}$ atoms) interactions are the most favorable in terms of energy though small differences are usually obtained. ${ }^{89,90}$ As our main purpose is to determine the switch of conductivity and not the real nature of $\mathrm{Au}-\mathrm{S}$ interaction, we selected for simplicity the pyramid configuration. Although tilted structures are possible, we only considered configurations in which the molecule was placed perpendicular to the electrode surface above a threefold facecentered-cubic (fcc) hollow site, i.e., those above a threefold site beneath which there is no Au atom in the second layer but with an $\mathrm{Au}$ atom in the third layer. An additional gold layer was included in one of the electrodes to ensure that the two S-Au connections were in similar fcc sites. Moreover, in all the devices, we fixed the orientation of the central ring to intersect the Au face-centered-cubic triangular faces. This assumption simplifies the problem because it is known that in these systems the conductivity depends on the intersection of the plane of the molecule in the Au face-centered-cubic triangular faces. ${ }^{91}$

We used molecule TPS-on to find the optimal S-Au distance in the pyramidal configuration for all TPS-based devices. To this end, we fully relaxed the supercell, gradually increasing the electrode distance until forces were less than $0.04 \mathrm{eV} / \AA$, using the Spanish Initiative for Electronic Simulations with Thousands of Atoms (SIESTA) code. ${ }^{92}$ The geometry of the supercell was optimized at DFT level with Perdew-BurkeErnzerhof generalized-gradient approximation (GGA-PEB) functional with a double- $\zeta$ plus polarization basis set for the organic molecule and gold atoms, with a 5-mRy energy-shift parameter, and a $5 \times 5$ Monkhorst-Pack grid for electronic structure calculations. During these calculations, core electrons and nuclei are replaced by Troullier-Martin's normconservative pseudopotentials. ${ }^{93}$ For the sake of simplicity, we selected an initial $\mathrm{Au}$-Au distance of $2.88 \AA$ in bulk $\mathrm{Au}$ and kept the $\mathrm{Au}$ layers fixed during the optimization process. This is a reasonable assumption due to the low distortion of the Au arrangement expected. ${ }^{94-96}$ While SIESTA supports the spin-polarized treatment of electrons, taking into account the nature of our system we employed only unpolarized calculations in this study. With these initial conditions we found that a distance from sulphur to the Au contact site of $2.25 \AA$, corresponding to an S-Au distance of $2.82 \AA$, presents a minimum energy. This value is close to other values described in similar Au-S arrangements. ${ }^{97-99}$ For ETP-on, the minimum energy was obtained with a slightly shorter S-Au bond length of $2.68 \AA$.

Once the $\mathrm{S}-\mathrm{Au}$ distance is determined, we know the electrode distance for the on devices, this being $19.86 \AA$ for TPS-on and $24.52 \AA$ for ETP-on. For the rest of the sulphurcontaining devices (off states TP-off, on states ETPF-on, ETPNO2-on, ETPOMe-on, and all off states ETP), in order to mimic a real device with a fixed length, we considered that their electrode separations were also those corresponding to TPS-on and ETP-on. All were fully relaxed, yielding almost symmetric devices. The only exceptions were TPS-off devices, which presented two different distances from sulphur to the contact site $(2.15$ and $2.30 \AA)$. It is worth noting that the linearity was almost conserved (TPS-off $1,176.10^{\circ}$; TPCN-off $1,177.50^{\circ}$ ).

The nitrile and isonitrile functionalized devices TPCN-on and TPNC-on were set up following similar reasoning. In this case, we placed the nitrogenated linker group in the top configuration. The optimal contact distances for TPCN-on and TPNC-on were 2.25 and $2.12 \AA$, respectively. Having established these distances, we set the rest of the devices.

With the optimized devices TP and ETP we carried out computational studies combining DFT and the nonequilibrium Green's-function scattering approach provided by a Quantum Wise software package ${ }^{100}$ to calculate the electron transport properties of our proposed molecular fuses. We used the GGA-PEB functional with the double- $\zeta$ plus polarization basis set for the organic molecule and the single- $\zeta$ plus polarization basis set for the Au electrodes. A $3 \times 3$ MonkhorstPack grid for electronic structure calculations and a $6 \times 6 \mathrm{~K}$ sampling in the transmission spectra with a 5-mRy energy-shift parameter were selected, based on convergence criteria and computational cost. The values of these three parameters are well tested in similar studies. ${ }^{101}$

Current $(I)$ was calculated as a function of the voltage using the Landauer formula,

$$
I=\frac{2 q}{h} \int_{0}^{\infty} T(E)\left[f\left(E-\mu_{L}\right)-f\left(E-\mu_{R}\right)\right] d E,
$$

where $T(E)$ is the transmission through the system as a function of the energy, $f$ is the Fermi-Dirac distribution function, $q$ is the charge of the electron, $h$ is Planck's constant, and $\mu_{\left.L_{(} R\right)}$ are the Fermi level at the applied voltage at the left (right) contact. When an external voltage $V$ is applied, the right voltage is $V / 2$ and the left contact voltage is set to $-V / 2$.

\section{RESULTS AND DISCUSSION}

\section{A. Influence of the anchoring groups}

In Fig. 4 we show a comparison between the transmission spectra of the three TP devices at low bias $(\Delta V \approx 0)$. The transport properties of the devices at low (operational) voltage $(V)$ are related to the nature of the transmission function at $E_{f} \pm V / 2, E_{f}$ being the Fermi level of gold. Thus we are interested mainly in the proximities of such a level, taking into account that molecular electronic devices are expected to work under a relatively low voltage (less than $1 \mathrm{~V}) .{ }^{102}$ However, for the sake of completeness, we show a wide voltage window of $4 \mathrm{eV}$ around the Fermi level in the figure. 


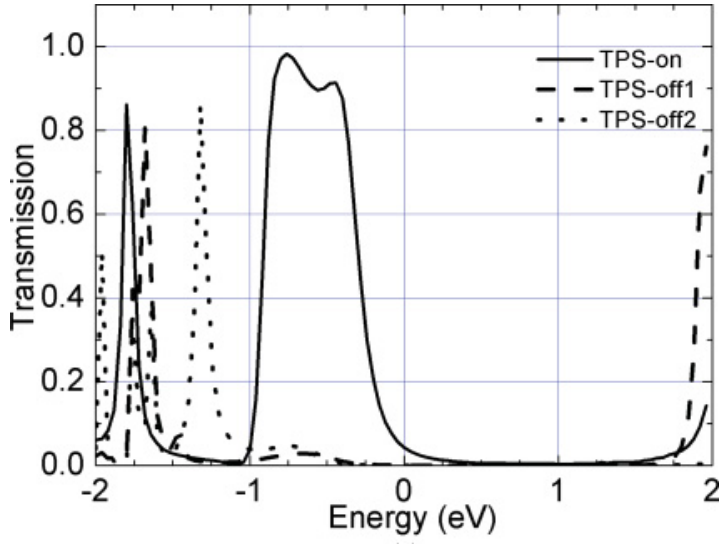

(a)

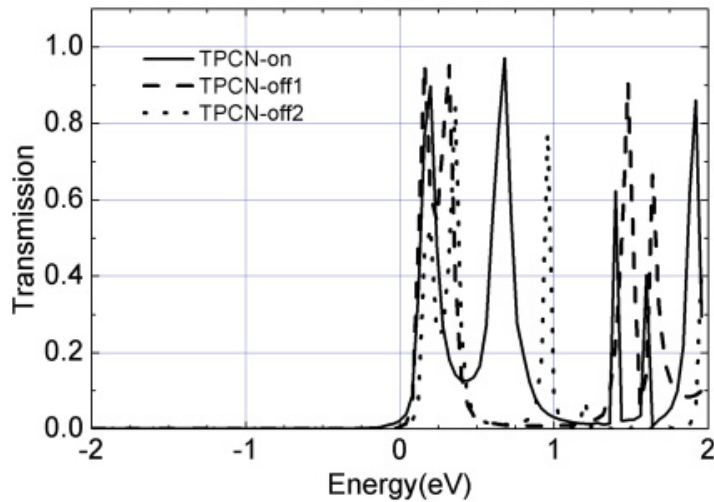

(b)

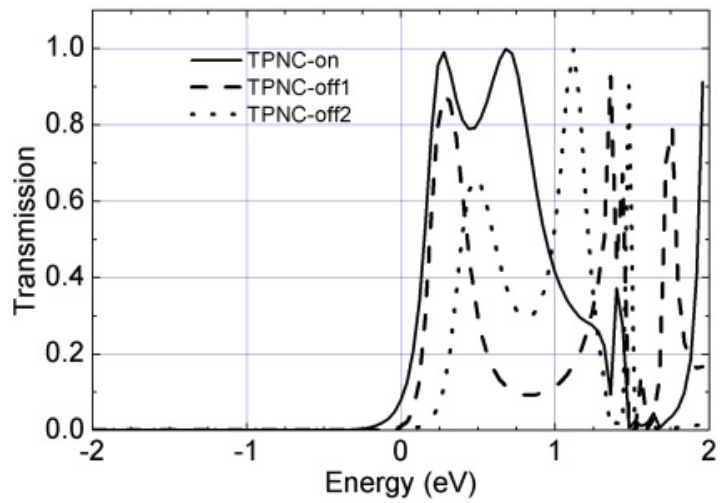

(c)

FIG. 4. (Color online) Transmission coefficients of TP devices at low bias.

When sulphur is used as linker, we can observe broad peaks, which correspond to a strong-coupling regime [Fig. 4(a)]. The main characteristic is that the transmission coefficients near the Fermi energy corresponding to the on device TPS-on are higher than those corresponding to off TPS-off 1 and TPS-off 2 devices. This is related to the presence of a broad, almost transparent resonance from -0.28 to $-0.94 \mathrm{eV}$ for the TPS-on device. An eigenchannel analysis showed that only one channel is open throughout the energy range studied $\left(E_{f} \pm 2.0 \mathrm{~V}\right)$ in the three devices. Molecular projected self-consistent Hamiltonian (MPSH) analysis is a useful tool to clarify the role of the molecule in the transport behavior of the global system, relating or not to the transmission peaks with the molecule. Such analysis is carried out projecting the self-consistent

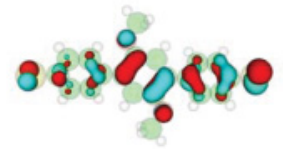

(a)

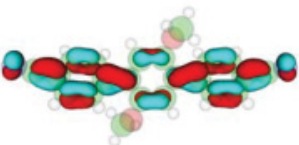

(c)

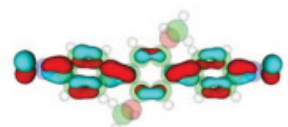

(e)

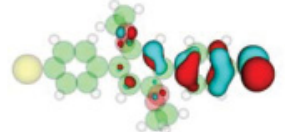

(b)

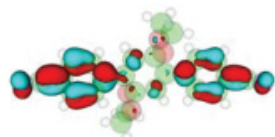

(d)

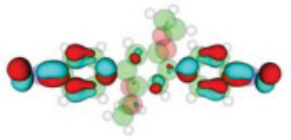

(f)
FIG. 5. (Color online) Isosurface plots of the MPSH wave functions for TPS devices at low bias $(V=0)$ at different energies: (a) HOMO level of TPS-on device at $-0.42 \mathrm{eV}$; (b) HOMO level of TPS-off 2 device at $-0.67 \mathrm{eV}$; (c) LUMO level of TPCN-on device at $0.14 \mathrm{eV}$; (d) LUMO level of TPCN-off 2 device at $0.14 \mathrm{eV}$; (e) LUMO level of TPNC-on device at $0.11 \mathrm{eV}$; (f) LUMO level of TPNC-off2 device at $0.24 \mathrm{eV}$.

Hamiltonian onto the atoms in the central region, and then diagonalized to produce an energy spectrum. This can be used to show how the molecular levels for a molecule are affected when placed between the two electrodes. ${ }^{100}$

Consequently with the above-mentioned results, a MPSH analysis of the TPS-on device showed that this broad transmission peak is derived from the presence of the HOMO level at $-0.42 \mathrm{eV}$ (see Fig. 5) and the HOMO-1 level at $-0.89 \mathrm{eV}$. Moreover, we observed a high delocalized wave function of HOMO and HOMO-1 levels in the MPSH isosurface plots for the TPS-on device. Therefore a good connection between the metallic electrodes is achieved, maximizing the transmission coefficients.

Predicting the on/off switch voltage in solid state is not a simple task because of the broadening of the molecular levels due to the interaction with the electrodes and the alignment of such levels toward the Fermi energy of the electrode. In a simple approximation, the oxidation reaction for these substrates is expected to occur when the difference of voltage centered in the Fermi energy of the electrode includes the broadened HOMO level, being the maximum probability of such an on/off switch at the center of the broadened HOMO level. Therefore an estimated oxidation potential (approximately $0.5-0.9 \mathrm{~V}$ ) is predicted for such structures with sulphur/gold junctions. However, DFT usually overestimates HOMO values and higher oxidation potentials would be expected in real experiments. ${ }^{103}$ This on/off voltage value is important because it limits the working voltage, which must always be smaller in order for the device to work properly. All these assumptions are limited by the fact that in this work we are considering only coherent transport in the final on and off states and not the inherent dynamic process of the switching event, which would be related to hopping mechanisms. In that 
case, instant charges would be developed in the molecule and probably the transmission spectrum would change. Although the precise mechanism of on/off transformation is not known even in homogeneous environments, previous calculations suggest that the global geometry of the system is retained during the switching event despite the generation of charge in the molecule. ${ }^{38}$

In TPS-off 1 and TPS-off 2 devices the electron transport is also dominated by broad peaks (TPS-off $1,-0.52$ to $-0.82 \mathrm{eV}$; TPS-off $2,-0.52$ to $-1.0 \mathrm{eV}$ ) but with much lower transmission coefficients. In this case, the low transmission values suggest an unfavorable mixing between electronic levels of the metallic electrodes at these energies. A similar MPSH analysis showed that the molecule has energy levels at these energies, but they are highly localized in only half of it, as we initially proposed, based on the insulating character of type-II structures. ${ }^{104} \mathrm{Al}-$ though the HOMO and HOMO-1 levels should be degenerated in TPS-off 1 and TPS-off 2 devices, we commented previously that they are asymmetrically joined to the electrodes and this asymmetry is revealed in the corresponding alignment of such levels. This geometrical asymmetry has no significant consequences in the transport mechanism but will provoke asymmetric $I / V$ curves.

It could be argued that the disconnection derives from differences in torsion angles in the three devices but this is not the sole reason: Device TPS-off $1\left(16.0^{\circ}\right)$ presents a lower torsion angle than TPS-on $\left(25.9^{\circ}\right)$. Nevertheless, if we compare off devices TPS-off 1 and TPS-off2, the expected relationship between the torsion angle and the conductivity is recovered (TPS-off $1,16.0^{\circ}$, TPS-off $2,42^{\circ}$ ).

The use of nitrogenated linkers changed the profile of the transmission spectra. In these cases, the conduction is LUMO controlled. ${ }^{95,96}$ We can also observe that sharper peaks are obtained in the case of nitrile $(-\mathrm{CN})$ containing devices TPCN because a weaker interaction between such groups and gold surfaces is expected. As in the case of TPS, only one eigenchannel is open in the voltage window studied. Moreover, the maximum of the transmission spectra is related to the presence of energy levels with a high contribution from the original organic molecule (TPCN-on, $0.14,0.63 \mathrm{eV}$; TPCNoff 1, 0.12, 0.28 eV; TPCN-off2, 0.14, $0.31 \mathrm{eV}$; TPNC-on, 0.11, $0.52 \mathrm{eV}$; TPNC-off1. 0.10, 0.22 eV; TPNC-off 2, 0.24, $0.34 \mathrm{eV}$ ). The main difference from TPS devices is the relatively high transmission coefficients corresponding to the off structures. The MPSH isosurface plots for TPS, TPCN, and TPNC devices showed the reason for this behavior. TPCN and TPNC LUMO levels are delocalized throughout the entire molecule, because the decoupling of the two subunits in the off states is inefficient. Another difference compared with the TPS-on device is the expected on/off switch voltage, which is related to the HOMO level. In this case, such levels are located at $-2.61 \mathrm{eV}$ for the TPCN-on structure and $-2.56 \mathrm{eV}$ for the TPNC-on structure. In this situation a nondesired on/off switch voltage of up to 6 $\mathrm{V}$ would be required to trigger the switching event.

Current can be also calculated. As we commented earlier, we are mainly interested in the low bias region because real operating systems will work under relatively low voltage. A voltage window of $1.5 \mathrm{~V}$ was therefore selected, taking into account also the experimental value of the oxidation peaks of terphenyls in solution. ${ }^{38}$ The currents calculated at different

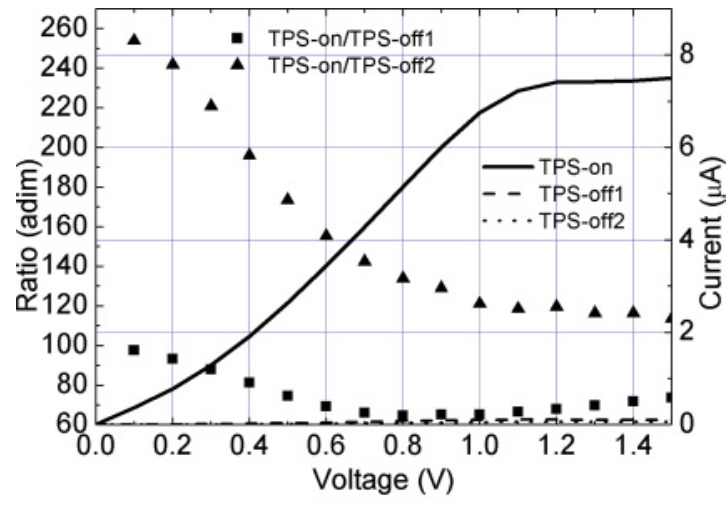

(a)

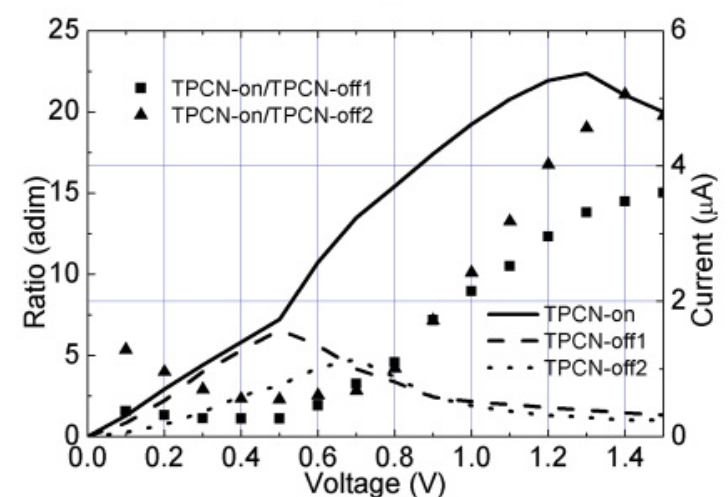

(b)

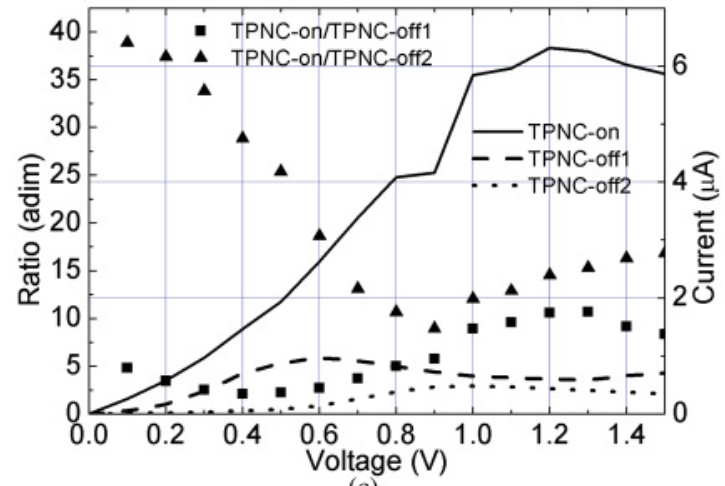

(c)

FIG. 6. (Color online) Current-voltage and switching values as voltage function.

voltages for TP devices are shown in the corresponding $I-V$ curves of Fig. 6.

According to the transmission spectra, the conductivity of conducting TP-on structures is higher than that of insulating TP-off structures. This fact is especially significant for sulphur bonded structures. Nevertheless, devices with similar length and structure, such as TP-off 1 and TP-off 2 , showed significant differences in conductivity, probably due to the different torsion angle values. This feature is worth noting because the torsion angle seems to be controlled by the nature of the side chains. Thus molecular fuses containing other more elaborated side chains might present a double switching process: a disconnection in the $\pi$-conjugated system and a higher torsion angle.

The $I / V$ curves are highly symmetric with the exception of the off structures of the TPS-on device, owing to the previously 
mentioned slight asymmetry in the anchoring groups. Even in this limit the differences in current are less than $7 \%$ and for the sake of clarity we have only shown the $I / V$ curve in the forward direction in all cases. We can observe high $d I / d V$ values at voltages lower than $1 \mathrm{~V}$, which belong to electrons with energies corresponding to the maxima of the transmission functions. Negative differential conductance (NDC) was also observed in TPCN and TPNC devices. This fact is assigned to misalignments between the Fermi level of the electrodes and the molecular levels derived from the presence of voltage.

We have estimated the on/off switch ratios from 97 at $0.1 \mathrm{~V}$ to 71 at $1.5 \mathrm{~V}$ for the TPS-on/TPS-off1 devices, and 253 at $0.1 \mathrm{~V}$ to 116 at $1.5 \mathrm{~V}$ for the TPS-on/TPS-off 2 devices. Lower values were obtained for the TPCN and TPNC devices. Thus, for example, a maximum value of 21 at $1.4 \mathrm{~V}$ was obtained for the TPCN-on/TPCN-off 2 pair and a maximum value of 38 at $0.1 \mathrm{~V}$ for the TPNC-on/TPNC-off2 pair. The TP-off2 structures always show the best switching values, taking into account that they present higher torsion angles.

\section{B. Influence of the substituents}

The substituents of ETP structures were selected to change the electronic richness of the key aromatic subunit. ${ }^{105,106}$ This may have two consequences: (i) a change in the oxidation potential of the on state and (i) a change in the alignment of the molecular energy levels with the Fermi level of gold, which is essential for electron transport. The first is interesting, since it allows the selection of molecular fuses with different on/off switch voltages depending on the substituents. The second is obviously related to the efficiency of the device. Taking into account that the better switching profile was obtained with a sulphur atom as a linker, the study focused on structures containing such a linker. Concerning the substituents, in addition to model systems ETP-on and ETP-off, we selected devices with two common deactivating (i.e., electron-withdrawing) groups such as fluorine (ETPF-on and ETPF-off, $R=\mathrm{F}$ ) and nitro (ETPNO2-on and ETPNO2-off, $R=-\mathrm{NO}_{2}$ ), and one prototypical activating (i.e., electron releasing) group such as the methoxy group (ETPOMe-on and ETPOMe-off, $R=$ OMe). ${ }^{107}$ Although fluorine is the least deactivating group among the halide family, it presents the least atomic volume and, consequently, the least distortion in the parent structure.

The transmission spectra of the ETP on devices, shown in Fig. 7, have broad, almost transparent resonances from -0.3 to $-1.0 \mathrm{eV}$, similar to the previously described TPS-on device. The first maximum is again correlated with the presence of a MPSH level (ETP-on, -0.46 eV; ETPF-on, -0.52 eV; ETPNO2-on, -0.70; ETPOMe-on, $-0.40 \mathrm{eV}$ ) (Fig. 8). The differences in energy of such MPSHs between ETP-on and the modified-ETP-on structures should depend on the activating and deactivating effects of the substituents in the aromatic ring. Nevertheless, if we consider the ETP-on device as a reference, we could only observe a significant decrease in the energy of the HOMO level of the ETPNO2-on device $(0.24 \mathrm{meV})$. The other variations of $0.06 \mathrm{meV}$ are well within error limits in the DFT calculations and cannot be certainly attributed to the electronic effects of the substituents.

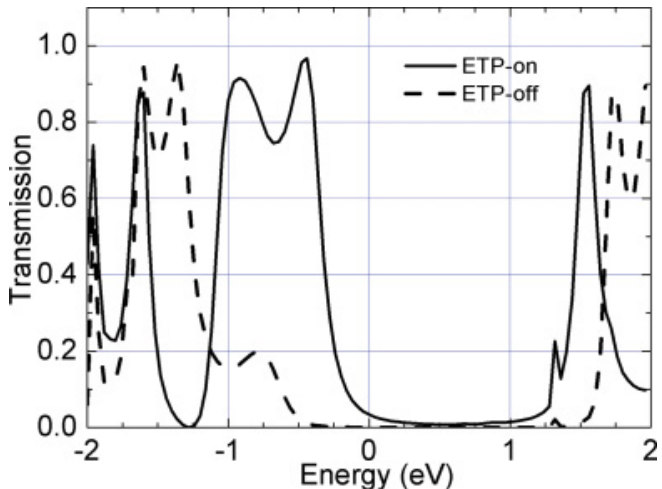

(a)

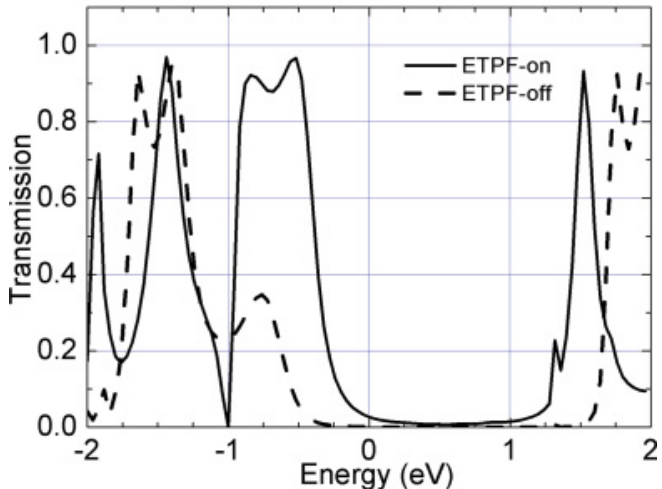

(b)

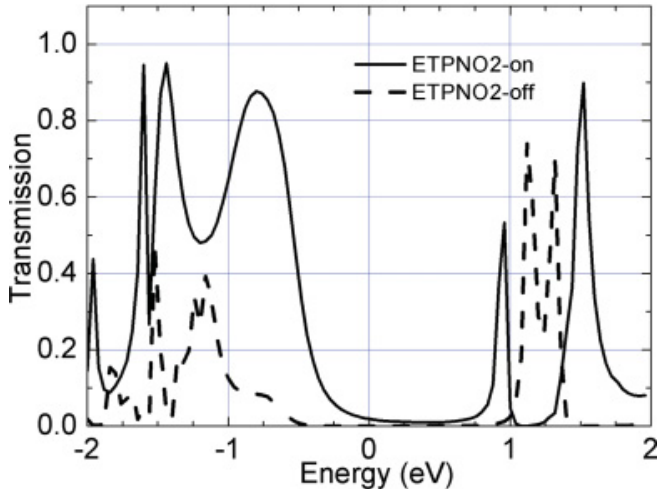

(c)

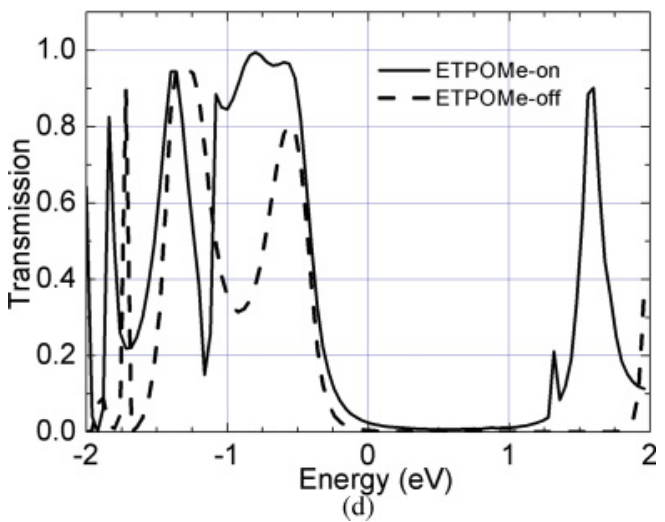

FIG. 7. (Color online) Transmission spectra of ETP devices at low bias.

Concerning the off devices, the lowest transmission coefficients at low bias were obtained with the ETPNO2off device, which includes the highest deactivating group 


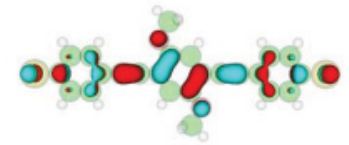

(a)

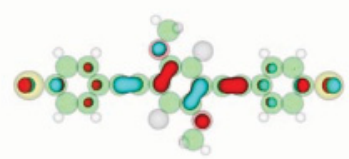

(c)

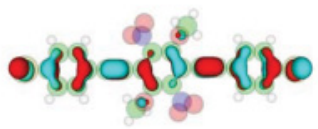

(e)

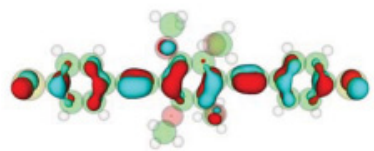

(g)

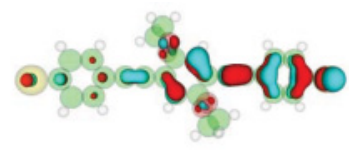

(b)

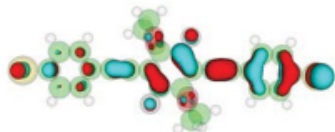

(d)

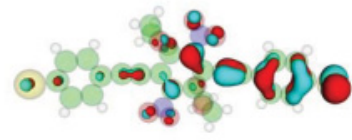

(f)

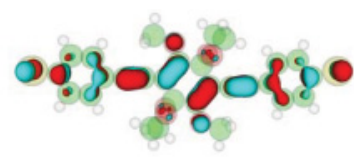

(h)
FIG. 8. (Color online) Isosurface plots of the MPSH wave functions for ETP devices at low bias $(V=0)$ at different energies: (a) HOMO level of ETP-on device at $-0.46 \mathrm{eV}$; (b) HOMO level of ETP-off device at $-0.76 \mathrm{eV}$; (c) HOMO level of ETPF-on device at $-0.52 \mathrm{eV}$; (d) HOMO level of ETPF-off device at $-0.74 \mathrm{eV}$; (e) HOMO level of ETPNO2-on device at $-0.70 \mathrm{eV}$; (f) HOMO level of ETPNO2-off device at $-0.71 \mathrm{eV}$; (g) HOMO level of ETPOMe-on device at $-0.40 \mathrm{eV}$; (h) HOMO level of ETPOMe-off device at $-0.49 \mathrm{eV}$.

studied. Consequently, the highest transmission coefficients correspond to the ETPOMe-off device, with the activating -OMe group. The fluorine-containing device ETPF-off and model device ETP-off showed intermediate values, with that corresponding to ETPF-off being slightly higher. An increase of the electron density in the off structures seems to derive an inefficient disconnection between the two subunits. The MPSH analysis of ETP devices demonstrates this fact. Two MPSH levels are present within the voltage window in all off ETP devices (ETP-off, -0.76 and $-0.92 \mathrm{eV}$; ETPF-off, -0.74 and $-0.94 \mathrm{eV}$; ETPNO2-off, -0.71 and $-0.81 \mathrm{eV}$; ETPOMe-off, -0.49 and $-0.73 \mathrm{eV})$. The nondegeneracy of HOMO and HOMO-1 levels in off ETP devices again reveals small asymmetries in the geometry of the device. Significant differences can be appreciated in the isosurface plots of the corresponding MPSH. The on ETP structures present a fully conjugated orbital while off structures are efficiently disconnected only in nitro- (ETPNO2-off) and hydrogen(ETP-off) substituted devices.

The $I-V$ curves [Fig. 9(a)] and switching ratios [Fig. 9(b)] at different voltages were calculated for ETP-on and ETPoff devices. From a practical point of view, the ETPNO2on/ETPNO2-off pair presents the best switching profile at low bias with on/off switch ratios ranging from 636 at $0.1 \mathrm{~V}$ to 52 at $1.5 \mathrm{~V}$. Interestingly, useful ratios can also be obtained using

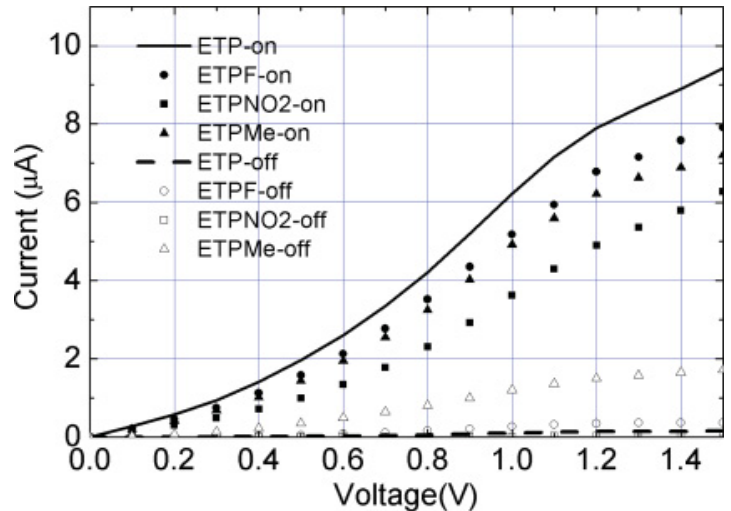

(a)

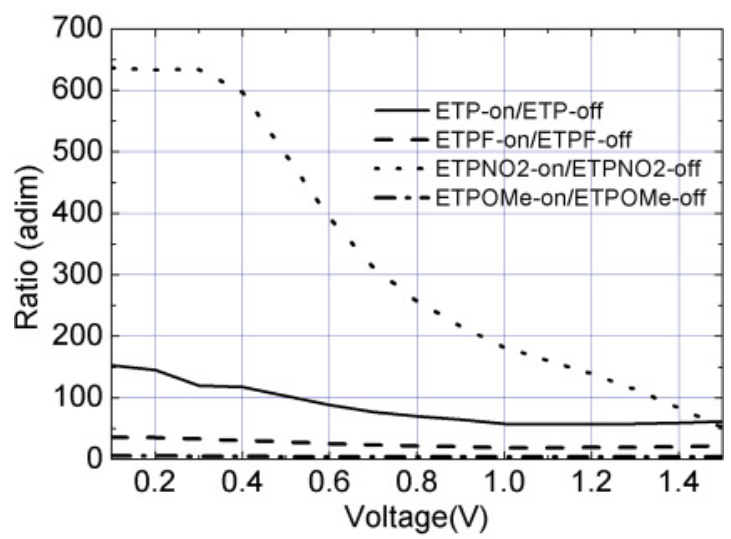

(b)

FIG. 9. (Color online) Current-voltage curves and switching values of ETP pairs as voltage function.

the simple unsubstituted ETP-on/ETP-off pair (152 at $0.1 \mathrm{~V}$ and 61 at $1.5 \mathrm{~V})$.

\section{CONCLUSIONS}

In summary, we have presented a study of the potential application of dialkoxybenzenes/quinone bisketal pairs as molecular fuses owing to their unique features, which could be of interest in the field of nanoelectronics. It is also worth noting that closely related structures have been synthesized recently, confirming some of the key predicted properties. Besides their remarkable geometrical behaviors we described in this paper interesting on/off switch ratios using in the modeling of the system SIESTA and Quantum Wise codes. The best on/off switch ratios were obtained when sulphur atoms were used as linkers between the molecule and the electrodes (TPS and ETP). In these particular cases the transmission spectra correspond to a system in which the molecular conductivity is HOMO controlled. Interestingly, in this regimen the on/off switch event could take place at a convenient voltage. With respect to the effect of the electron richness on the on/off switch ratio we found that strongly deactivating groups $\left(-\mathrm{NO}_{2}\right)$ highly improve it. Thus, for example, the on/off switch ratio ranged from 636 for deactivated device ETPNO2, being only 7 for activated ETPOMe for the same voltage $(0.1 \mathrm{~V})$. As the on/off switch voltage is controlled by the position of the HOMO 
level and it does not substantially change by the presence of different substituents, we predict a relatively small influence of such substituents on the on/off switch voltage. Therefore we can conclude that molecules presenting sulfur-based groups as linkers and electron withdrawing groups in the central aromatic ring would be interesting candidates as prototypical nanofuses for experimental measures. Although the present calculations suggest the viability of our proposal, a real device should increment the on/off switch ratio from the calculated two orders of magnitude to at least six orders of magnitude. Based on these precedents new calculations of a second generation of nanofuses are underway.

\section{ACKNOWLEDGMENTS}

We thank the Regional Government of Andalucía for financial support (Projects No. P06-FQM-01726 and No. P09-FQM-04571), the "Centro de Supercomputación de la Universidad de Granada," and the "Centro de Computación Científica-UAM" for computation time. The authors are also grateful to the Spanish Secretaría de Estado de Universidades e Investigación, Ministerio de Educación y Ciencia, for financial support within research projects TEC2007-66812 and TEC2010-16211. N.F. thanks the Regional Government of Andalucía for her research contract, and LAC thanks the University of Granada for his research contract. *rbolivar@ugr.es

†fmgomez@ugr.es

†lac@ugr.es

§mcuerva@ugr.es

${ }^{1}$ C. Joachim and M. A. Ratner, Proc. Nat. Acad. Sci. USA 102, 8801 (2005).

${ }^{2}$ F. Chen, J. Hihath, Z. Huang, X. Li, and N. J. Tao, Annu. Rev. Phys. Chem. 58, 535 (2007).

${ }^{3}$ L. A. Bumm, J. J. Arnold, M. T. Cygan, T. D. Dunbar, T. P. Burgin, L. Jones, D. L. Allara, and J. M. P. S. Weiss, Science 271, 1705 (1996).

${ }^{4}$ J. Park, A. N. Pasupathy, J. I. Goldsmith, C. Chang, Y. Yalsh, J. R. Petta, M. Rinkoski, J. P. Sethna, H. D. Abruña, P. L. Mceuen, and D. C. Ralph, Nature (London) 417, 722 (2002).

${ }^{5}$ W. Liang, M. P. Shores, M. Bockrath, J. R. Long, and H. Park, Nature (London) 417, 725 (2002).

${ }^{6} \mathrm{~B} . \mathrm{Xu}$ and N. J. Tao, Science 301, 1221 (2003).

${ }^{7}$ T. Dadosh, Y. Gordin, R. Krahne, I. Khivrich, D. Mahalu, V. Frydman, J. Sperling, A. Yacoby, and I. Bar-Joseph, Nature (London) 436, 677 (2005).

${ }^{8}$ X. Chen, Y.-M. Jeon, J.-W. Jang, L. Quin, F. Huo, W. Wei, and C. A. Mirkin, J. Am. Chem. Soc. 130, 8166 (2008).

${ }^{9}$ G. Sedghi, K. Sawada, L. J. Esdaile, M. Hoffmann, H. L. Anderson, D. Bethell, W. Haiss, S. J. Higgins, and R. J. Nichols, J. Am. Chem. Soc. 130, 8582 (2008).

${ }^{10}$ D. Kockmann, B. Poelsema, and H. J. W. Zandvliet, Nano Lett. 9, 1147 (2009).

${ }^{11}$ L. Lafferentz, F. Ample, H. Yu, S. Hecht, C. Joachim, and L. Grill, Science 323, 1193 (2009).

${ }^{12}$ X. Chen, A. B. Braunschweig, M. J. Wiester, S. Yeganeh, M. A. Ratner, and C. A. Mirkin, Angew. Chem. Int. Ed. 48, 5178 (2009).

${ }^{13}$ T. Ikeda, N. Aratani, and A. Osuka, Chem. Asian J. 4, 1248 (2009).

${ }^{14}$ C. Liu, D. Walter, D. Neuhauser, and R. Baer, J. Am. Chem. Soc. 125, 13936 (2003)

${ }^{15}$ J. S. Vrettos, D. H. Stewart, J. C. de Paula, and G. W. Brudvig, J. Phys. Chem. B 103, 6403 (1999).

${ }^{16}$ P. Faller, A. Pascal, and A. W. Rutherford, Biochemistry 40, 6431 (2001).

${ }^{17}$ J. P. Collman, N. K. Devaraj, R. A. Decreau, Y. Yang, Y.-L. Yan, W. Ebina, T. A. Eberspacher, and E. D. Chidsey, Science 315, 1565 (2007).
${ }^{18}$ K. Szacilowski, Chem. Rev. 108, 3481 (2008).

${ }^{19}$ B. A. Mantooth and P. S. Weiss, Proc. IEEE 91, 1785 (2003).

${ }^{20}$ K. Seo, A. V. Konchenko, J. Lee, G. S. Bang, and H. Lee, J. Am. Chem. Soc. 130, 2553 (2008).

${ }^{21}$ E. Green, J. W. Choi, A. Boukai, Y. Bunimovich, E. JohnstonHalprin, E. DeIonno, Y. Luo, B. A. Sheriff, K. Xu, Y. S. Shin, H.-R. Tseng, J. F. Stoddart, and J. R. Heath, Nature (London) 445, 414 (2007).

${ }^{22}$ A. C. Whalley, M. L. Steigerwald, X. Guo, and C. Nuckolls, J. Am. Chem. Soc. 129, 12590 (2007).

${ }^{23}$ D. R. Henton, R. L. McCreery, and J. S. Swenton, J. Org. Chem. 45, 369 (1980).

${ }^{24}$ J. S. Swenton, Acc. Chem. Res. 16, 74 (1983).

${ }^{25} \mathrm{P}$. Maksymovych and J. T. Yate, J. Am. Chem. Soc. 130, 7518 (2008).

${ }^{26}$ J. Fortage, E. Göransson, E. Blart, H.-C. Becker, L. Hammaeström, and F. Odobel, Chem. Commun. 4629 (2007).

${ }^{27}$ C. Wang, A. S. Batsanov, M. R. Bryce, G. J. Ashwell, B. Urasinska, I. Grace, and C. J. Lambert, Nanotechnology 18, 1 (2008).

${ }^{28}$ C. Wang, A. S. Batsanov, M. R. Bryce, and I. Sage, Org. Lett. 6, 2181 (2006).

${ }^{29}$ C. Atienza, N. Matin, M. Wielopolski, N. Haworth, T. Clark, and D. M. Guldi, Chem. Commun. 3202 (2006).

${ }^{30}$ J. Fortage, J. Boixel, E. Blart, L. Hammaeström, H. C. Becker, and F. Odobel, Chem. Eur. J. 14, 3467 (2008).

${ }^{31}$ C. Engtrakul and L. R. Sita, Organometallics 27, 927 (2008).

${ }^{32}$ G. J. Ashwell, B. Urasinska, C. Wang, M. R. Bryce, I. Grace, and C. J. Lambert, Chem. Commun. 4706 (2006).

${ }^{33}$ B. L. Chenard, M. J. Manning, P. W. Raynolds, and J. S. Swenton, J. Org. Chem. 45, 378 (1980).

${ }^{34}$ M. P. Capparelli and J. S. Swenton, J. Org. Chem. 52, 5360 (1987).

${ }^{35}$ T. M. Swager, M. M. Rock, and R. H. Grubbs, New Polym. Mater. 2, 1 (1990).

${ }^{36}$ M. Mayor, C. von Hänish, H. B. Weber, J. Reichert, and D. Beckmann, Angew. Chem. Int. Ed. 41, 1183 (2002).

${ }^{37}$ G. A. Jeffrey, J. Buschman, C. W. Lehmann, and P. Luger, J. Am. Chem. Soc. 110, 7218 (1988).

${ }^{38}$ N. Fuentes, L. Álvarez de Cienfuegos, A. Parra, D. ChoquesilloLazarte, J. M. García-Ruiz, M. L. Marcos, E. Buñuel, M. Ribagorda, M. C. Carreño, D. J. Cárdenas, and J. M. Cuerva, Chem. Commun. 47, 1586 (2011).

${ }^{39}$ D. James and J. M. Tour, Chem. Mater. 16, 4423 (2004). 
${ }^{40}$ B. Ulgut and H. D. Abruña, Chem. Rev. 108, 2721 (2008).

${ }^{41}$ A. Nitzan, Annu. Rev. Phys. Chem. 52, 681 (2001).

${ }^{42}$ M. Koentopp, C. Chang, K. Burke, and R. Car, J. Phys.: Condens. Matter 20, 083203 (2008).

${ }^{43}$ Y. Hu, Y. Zhu, H. Gao, and H. Guo, Phys. Rev. Lett. 95, 156803 (2005).

${ }^{44}$ C. Li, I. Povelob, T. Wandlowski, A. Bagrets, A. Arnold, and F. Evers, J. Am. Chem. Soc. 130, 318 (2008).

${ }^{45}$ H. Basch, R. Cohen, and M. A. Ratner, Nano Lett. 5, 1668 (2005).

${ }^{46}$ K.-H. Müller, Phys. Rev. B 73, 045403 (2006).

${ }^{47}$ F. Pauly, J. K. Viljas, J. C. Cuevas, and G. Schön, Phys. Rev. B 77, 155312 (2008).

${ }^{48}$ H. Cao, J. Jiang, J. Ma, and Y. Luo, J. Am. Chem. Soc. 130, 6674 (2008).

${ }^{49}$ S.-H. Ke, H. U. Baranger, and W. Yang, J. Chem. Phys. 126, 201102 (2007).

${ }^{50}$ A. J. Cohen, P. Mori-Sánchez, and W. Yang, Science 321, 792 (2008).

${ }^{51}$ S. Y. Quek, L. Venkataraman, H. J. Choi, S. G. Louis, M. S. Hybertsen, and J. B. Neaton, Nano Lett. 7, 3477 (2007).

${ }^{52}$ S. Martin, W. Haiss, S. J. Higgins, and R.J. Nichols, Nano Lett. 10, 2019 (2010).

${ }^{53}$ A. C. Whalley, M. L. Steigerwald, X. Guo, and C. Nuckolls, J. Am. Chem. Soc. 129, 12590 (2007).

${ }^{54}$ C. Zhang, M.-H. Du, H.-P. Cheng, X.-G. Zhang, A. E. Roitberg, and J. L. Krause, Phys. Rev. Lett. 92, 158301 (2004).

${ }^{55}$ S. S. Jang, Y. H. Jang, Y.-H. Kim, W. A. Goddard, A. H. Flood, B. W. Laursen, H.-R. Tseng, J. F. Stoddart, J. O. Jeppesen, J. W. Choi, D. W. Steuerman, E. Delonno, and J. R. Heath, J. Am. Chem. Soc. 127, 1563 (2005).

${ }^{56}$ A. Staykov, D. Nozaki, and K. Yoshizawa, J. Phys. Chem. C 111, 3517 (2007).

${ }^{57}$ J. Li, G. Speyer, and O. F. Sankey, Phys. Rev. Lett. 93, 248302 (2004).

${ }^{58}$ J. L. Palma, C. Cao, X.-G. Zhang, P. L. Krstic, J. L. Krause, and H.-P. Cheng, J. Phys. Chem. C 114, 1655 (2010).

${ }^{59}$ X. Li, J. Hihath, F. Chen, T. Masuda, L. Zang, and N. Tao, J. Am. Chem. Soc. 129, 11535 (2007).

${ }^{60}$ D. Dulic, S. J. van der Molen, T. Kudernac, H. T. Jonkman, J. J. D. de Jong, T. N. Bowden, J. van Esch, B. L. Feringa, and B. J. van Wees, Phys. Rev. Lett. 91, 207402 (2003).

${ }^{61}$ J. Tang, Y. Wang, J. E. Klare, G. S. Tulevski, S. J. Wind, and C. Nuckolls, Angew. Chem. Int. Ed. 46, 3892 (2007).

${ }^{62}$ M. Hasan, D. Bethell, and M. Brust, J. Am. Chem. Soc. 124, 1132 (2002).

${ }^{63}$ T. Ishida, W. Mizutani, N. Choi, U. Akiba, M. Fujihira, and H. Tokumoto, J. Phys. Chem. 104, 11680 (2000).

${ }^{64}$ M. Banarjee, R. Shukla, and R. Rathore, J. Am. Chem. Soc. 131, 1780 (2009).

${ }^{65}$ L. Vankataraman, J. E. Klere, C. Nuckolls, M. S. Hybertsen, and M. L. Stigerwald, Nature (London) 442, 904 (2006).

${ }^{66}$ R. L. McCreery, Chem. Mater. 16, 4477 (2004).

${ }^{67}$ A. Danilov, S. Kubatkin, S. Kafanov, P. Hedegard, N. StuhrHansen, K. Moth-Poulsen, and T. Bjornhold, Nano Lett. 8, 1 (2008).

${ }^{68}$ J. R. Quinn, F. W. Foss, L. Venkatamaran, and R. Breslow, J. Am. Chem. Soc. 129, 12376 (2007).

${ }^{69}$ L. Venkataraman, Y. S. Park, A. C. Whalley, C. Nuckolls, M. S. Hybertseen, and M. L. Steigerwald, Nano Lett. 7, 502 (2007).
${ }^{70}$ L. Venkatamaran, J. Klare, I. W. Tam, C. Nuckolls, M. S. Hybertsen, and M. L. Steigerwald, Nano Lett. 6, 458 (2006).

${ }^{71}$ C. Chu, J. A. Ayres, D. M. Stefanescu, B. R. Walker, C. B. Gorman, and G. N. Parsons, J. Phys. Chem. 111, 8080 (2007).

${ }^{72}$ J. M. Beebe, V. B. Engelkes, L. L. Miller, and C. D. Frisbie, J. Am. Chem. Soc. 124, 11268 (2002).

${ }^{73}$ M. Kiguchi, S. Miura, K. Hara, M. Sawamura, and K. Murakoshi, Appl. Phys. Lett. 89, 213104 (2006).

${ }^{74}$ J. Chen, L. C. Calvet, M. A. Reed, D. W. Carr, D. S. Grubisha, and D. W. Bennett, Chem. Phys. Lett. 313, 741 (1999).

${ }^{75}$ B. Kim, J. M. Beebe, Y. Jun, X.-Y. Zhu, and D. Frisbie, J. Am. Chem. Soc. 128, 4970 (2006).

${ }^{76}$ S. Yasuda, S. Yoshida, J. Sasaki, Y. Okutsu, T. Nakamura, A. Taninaka, O. Takeuchi, and H. Shigekawa, J. Am. Chem. Soc. 128, 7746 (2006).

${ }^{77}$ A. V. Tivanski, Y. He, E. Borguet, H. Liu, G. C. Walker, and D. Waldeck, J. Phys. Chem. B 109, 5398 (2005).

${ }^{78}$ T.-H. Park and M. J. Therien, Org. Lett. 9, 2779 (2007).

${ }^{79}$ S. Dixon and R. J. Whitby, Tetrahedron Lett. 47, 8147 (2006).

${ }^{80}$ Y.-V. Kervennic, J. M. Thijssen, D. Vammackelbergh, R. Dabirian, L. W. Jenneskens, C. A. van Walree, and H. S. van der Zant, J. Angew. Chem. Int. Ed. 45, 2540 (2006).

${ }^{81}$ Y. S. Park, A. C. Whalley, M. Kamenetska, M. L. Steigerwald, M. S. Hybertsen, C. Nuckolls, and L. Venkataraman, J. Am. Chem. Soc. 129, 15768 (2007).

${ }^{82}$ F. Chen, X. Li, J. Hihath, Z. Huang, and N. Tao, J. Am. Chem. Soc. 129, 15874 (2006).

${ }^{83}$ C. A. Martin, D. Ding, J. K. Sorensen, T. Bjomholm, J. M. van Ruitenbeek, and H. S. J. van der Sant, J. Am. Chem. Soc. 130, 13198 (2008).

${ }^{84}$ Y. S. Park, A. C. Whalley, M. Kamenetska, M. L. Steigerwald, M. S. Hybertsen, C. Nuckolls, and L. Venkataraman, J. Am. Chem. Soc. 129, 15768 (2007).

${ }^{85}$ J. R. Quinn, F. W. Foss, L. Venkataraman, M. S. Hybertsen, and R. Breslow, J. Am. Chem. Soc. 129, 6714 (2007).

${ }^{86}$ M. Mayor, H. B. Weber, J. Reichert, M. Elbing, C. von Hanisch, D. Beckmann, and M. Fischer, Angew. Chem. Int. Ed. 42, 5834 (2003).

${ }^{87}$ P. D. Jadzinsky, G. Calero, J. Ackerson, D. A. Bushnell, and R. D. Kornberg, Science 318, 430 (2007).

${ }^{88}$ P. Maksymovych and J. T. Yate, J. Am. Chem. Soc. 130, 7518 (2008).

${ }^{89}$ S. H. Ke, H. U. Baranger, and W. Yang, J. Am. Chem. Soc. 126, 15897 (2004).

${ }^{90}$ H. Grönbeck, A. Curioni, and W. Andreoni, J. Am. Chem. Soc. 122, 3839 (2000).

${ }^{91}$ D. Q. Andrews, R. P. Van Duyne, and M. A. Ratner, Nano Lett. 8, 1120 (2008).

${ }^{92}$ D. Sanchez-Portal, P. Ordejon, E. Artacho, and J. M. Soler, Int. J. Quantum Chem. 65, 453 (1997). For a complete description of the code, see [http://www.uam.es/siesta]

${ }^{93}$ N. Troullier and J. L. Martins, Phys. Rev. B 43, 1993 (1991).

${ }^{94}$ M. J. Ford, C. Masens, and M. B. Cortie, Surf. Rev. Lett. 13, 297 (2006)

${ }^{95}$ Y. Xue and M. A. Ratner, Phys. Rev. B 69, 085403 (2004).

${ }^{96}$ B. Kim, J. M. Beebe, Y. Jun, X.-Y. Zhu, and D. Frisbie, J. Am. Chem. Soc. 128, 4970 (2006).

${ }^{97}$ Y. Karzazi, X. Crispin, O. Kwon, J. L. Bredas, and J. Cornil, J. Chem. Phys. Lett. 387, 502 (2004). 
${ }^{98}$ M. Girlanda, I. Cacelli, A. Ferreti, and M. Macucci, J. Comp. Electron. 4, 87 (2005)

${ }^{99}$ C. Toher and S. Sanvito, Phys. Rev. B 77, 155402 (2008).

${ }^{100}$ Atomistix ToolKit version 2008.10, QuantumWise A/S [http://www.quantumwise.com]

${ }^{101}$ R. C. Hoft, M. J. Ford, and M. B. Cortie, Mol. Simul. 33, 897 (2007).

${ }^{102}$ International technology roadmap for semiconductors. Emerging devices, 2009. See also [http://www.itrs.net].
${ }^{103}$ C. D. Pemmaraju, T. Archer, D. Sánchez-Portal, and S. Sanvito, Phys. Rev. B 75, 045101 (2007).

${ }^{104}$ D. Q. Andrews, R. Cohen, R. P. Van Duyne, and M. A. Ratner, J. Chem. Phys. 125, 174718 (2006).

${ }^{105}$ H. Chen, J. Q. Lu, J. Wu, R. Note, H. Mizuseki, and Y. Kawazoe, Phys. Rev. B 67, 113408 (2003).

${ }^{106}$ L. Venkataraman, Y. S. Park, A. C. Whalley, C. Nuckolls, M. S. Hybertsen, and M. L. Steigerwald, Nano Lett. 7, 502 (2007).

${ }^{107}$ C. Hansch, A. Leo, and R. W. Taft, Chem. Rev. 91, 165 (1991). 